\title{
Código Europeo Contra el Cáncer y Salud Laboral. Actuación de la Enfermería del Trabajo
}

\author{
European Code Against Cancer and Health. Performance of Occupational Health \\ Nursing
}

\section{Jesús González Sánchez}

Servicio de Prevención de Riesgos Laborales. MC - Prevención Salamanca. España.

Recibido: 19-07-12

Aceptado: 08-08-12

\section{Correspondencia}

Jesús González Sánchez

MC - Prevención Salamanca

Plaza Mariseca, s/n. esquina Calle del Hornazo

37003 - Salamanca. España

E-mail: igonzalezsa@mc-prevencion.com

\section{Resumen}

El cáncer se ha convertido en una de las principales causas de morbi-mortalidad de la sociedad actual, afectando cada vez a un mayor número de personas y a un grupo de edades mucho más amplio, incluyéndose de forma importante la edad laboral.

El Código Europeo Contra el Cáncer, constituye una herramienta eficaz para el profesional de la salud laboral, teniendo aplicación dentro de las competencias específicas otorgadas al especialista en Enfermería del Trabajo, y facilitando la realización de estudios e investigaciones epidemiológicas, que permitan obtener una estrategia de actuación preventiva eficaz, ante múltiples factores de riesgo de cáncer laboral presentes en determinadas industrias, contribuyendo a una correcta vigilancia y control de la salud.

Med Segur Trab (Internet) 2012; 58 (228) 282-290

Palabras claves: Cáncer, Salud Laboral, prevención.

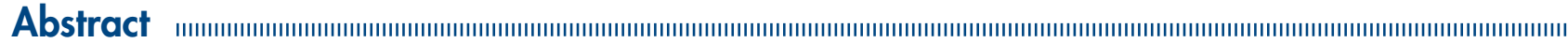

Cancer has become a major cause of morbidity and mortality in today's society, affecting more and more people and an age group much broader, including a significant working age. The European Code Against Cancer, is an effective tool for occupational health professional, having application within the powers granted to a specialist in Occupational Health Nursing, facilitating studies and epidemiological research that will generate a strategy for preventive action effectively to multiple risk factors for occupational cancer present in certain industries, achieving a proper monitoring and health surveillance.
\end{abstract}

Med Segur Trab (Internet) 2012; 58 (228) 282-290

Key words: Cancer, occupational health, prevention. 


\section{INTRODUCCIÓN}

El padecer algún tipo de enfermedad cancerígena, afecta cada vez a un mayor número de personas en edades tempranas, coincidiendo en muchos casos con su edad laboral. Los factores de riesgo presentes en el trabajo, son causa frecuente de morbilidad y mortalidad en la población. Se estima que más del 16\% de los procesos atendidos en centros de Atención Primaria de Salud, están probablemente relacionados con las condiciones de trabajo $^{1}$ ).

Gracias a la investigación y aparición de nuevas terapias, se ha producido un aumento en el número de personas que logran superar las enfermedades de origen cancerígeno y consiguen reincorporarse a su vida laboral anterior.

Los cánceres de origen profesional, son la fuente de información más antigua y a la vez más eficiente para el descubrimiento de agentes cancerígenos ${ }^{2,3}$. El control de la exposición laboral a factores de riesgo cancerígenos es factible, y si se realizan correctamente, pueden prevenir un considerable número de cánceres entre los trabajadores ${ }^{4}$.

En lo referente a la vigilancia y control del cáncer de origen laboral, debemos reflejar que, España es un país con una reciente normativa en lo que a la vigilancia de la salud de los trabajadores se refiere. En el capítulo IV de la ley General de Sanidad de $1986^{5}$, se establecen criterios fundamentales respecto a la vigilancia de la salud de forma global, cuyo desarrollo posterior permitirá alcanzar los objetivos de la prevención de los riesgos laborales y la promoción de la salud física y mental de los trabajadores. En dicho capítulo también se indica la necesidad de investigar las condiciones de trabajo, vigilar y promover la salud de los trabajadores, informarles y formarles adecuadamente, pero no se hace referencia clara a actividades concretas dirigidas a actuar en la vigilancia del cáncer laboral.

Con la aparición en 1995 de la ley de prevención de riesgos laborales ${ }^{6}$, se inicia una actividad de vigilancia y control de factores de riesgo para la salud mucho más detallada, incluyéndose de forma especial los relacionados con el cáncer laboral, y añadiéndose aspectos normativos específicos como el Real Decreto $665 / 1997^{7}$, sobre la protección de los trabajadores contra los riesgos relacionados con la exposición a agentes cancerígenos durante el trabajo.

En el año 2004, el gobierno se comprometió con los agentes sociales, fuerzas políticas y comunidades autónomas, a impulsar acciones tendentes a reducir la siniestralidad laboral y mejorar de forma continua los niveles de seguridad y salud en el trabajo. Esto se plasmó en el documento "Estrategia española en materia de Seguridad y Salud en el Trabajo 2007-2012" 8 .

En 1.985 nace el programa EUROPA CONTRA EL CÁNCER, con el objetivo de disminuir la mortalidad por esta causa. Dentro de este programa se desarrolla el Código Europeo Contra el Cáncer (CECC), documento de prevención en el que se presentan los principales factores de riesgo, síntomas de alarma y técnicas de diagnóstico precoz de la enfermedad.

La última revisión de este documento se presentó en Bruselas el 17 de Junio de 2003, y actualmente consta de once recomendaciones claves (Figura 1). 


\section{Figura 1. Código Europeo Contra el Cáncer}

1. No fume. Si no consigue dejar de fumar, no fume en presencia de no fumadores.

2. Evite la obesidad.

3. Realice alguna actividad física vigorosa todos los días.

4. Aumente su ingesta diaria de frutas, verduras y hortalizas variadas: coma al menos cinco raciones al día.

5. Si bebe alcohol, ya sea cerveza, vino o bebidas de alta graduación, modere el consumo a un máximo de dos consumiciones diarias, si es hombre, o a una, si es mujer.

6. Tome precauciones para evitar la exposición excesiva al sol. Consulte a su medico si nota un lunar que cambia de forma, tamaño o color, algún bulto o herida que no cicatriza.

7. Aplique de forma estricta la legislación destinada a prevenir cualquier exposición a sustancias carcinogénicas. Siga las instrucciones de seguridad y salud sobre el uso de estas sustancias que pueden causar cáncer. Respete las normas dictadas por las oficinas nacionales de protección radiológica.

Existen programas de salud pública que pueden prevenir el desarrollo de cánceres o aumentar la probabilidad de curar un cáncer:

8. Las mujeres a partir de los 25 años de edad deberían someterse a pruebas de detección precoz del cáncer de cuello de útero.

9. Las mujeres a partir de los 50 años de edad deberían someterse a pruebas de detección precoz del cáncer de mama.

10. Los hombres y las mujeres a partir de los 50 años de edad deberían someterse a pruebas de detección precoz de cáncer de colon.

11. Participe en programas de vacunación contra la infección por el virus de la hepatitis $\mathrm{B}$.

Es importante señalar que, pese a la situación de la crisis económica global que se está produciendo en los últimos años, y más en concreto en España, se deben de seguir llevando a cabo medidas relacionadas con la vigilancia y control del cáncer laboral, como es el caso de la utilización del Código Europeo Contra el Cáncer, ya que de no ser así, las consecuencias humanas y económicas que se podrían generar, serían fatales para la población trabajadora y el propio sistema nacional de salud.

Se estima que un 25 \% de los trabajadores en España están expuestos a carcinógenos en su lugar de trabajo, pero la magnitud real del cáncer por exposición laboral en nuestro país es desconocida ${ }^{9}$.

\section{OBJETIVOS}

Como objetivo general planteamos la demostración de la aplicación en el ámbito laboral y dentro de las competencias y funciones de la Enfermería del Trabajo, del Código Europeo Contra el Cáncer, como una herramienta sanitaria fácil de utilizar y efectiva hacia los trabajadores. 
Entre los objetivos específicos se encuentran:

1. Analizar la prestación de cuidados y atención dada al trabajador.

2. Valorar aspectos y situaciones que se benefician o potencian con la aplicación de este código, como la comunicación con el trabajador así como su colaboración en alguna toma de decisión y aspectos relacionados con su salud.

3. Intentar relacionar con su aplicación, una mejora de atención y mayor calidad de cuidados prestada al trabajador.

4. Facilitar el análisis y estudio de datos e información sanitaria, que favorezca la realización de estudios epidemiológicos y trabajos de investigación sobre el cáncer.

\section{MATERIAL Y MÉTODOS}

Para la realización de este trabajo, he realizado una búsqueda bibliográfica utilizando para ello material como: libros y revistas sobre Prevención de Riesgos Laborales, medicina laboral y normativa al respecto, así como una búsqueda documental en internet a través de bases de datos, referidas en la bibliografía, de rigor científico como:

- Index de Enfermería (Cuiden)

- Pubmed

- Cochrane Plus

- Medline Plus

- Enfispo

- Teseo

Dicha búsqueda en internet se ha realizado acotando a las voces de:

"Cancerígenos laborales".

“Cáncer y trabajo".

"Epidemiología y Cáncer".

“Código Europeo Cáncer”.

"Vigilancia de la Salud".

"Enfermedades profesionales".

\section{Aplicación de Código Europeo Contra el Cáncer, dentro de las competencias específicas de la Enfermería del Trabajo:}

En la Orden SAS/1348/2009, de 6 de mayo ${ }^{10}$, se aprueba y publica el programa formativo de la especialidad de Enfermería del Trabajo. Dentro de este programa se detallan unas competencias específicas (Figura 2), que el especialista en enfermería del Trabajo debe de ejercer en los servicios de prevención, tanto ajenos como propios, pudiendo utilizar el Código Europeo Contra el Cáncer, como una herramienta clave en la actividad preventiva del cáncer laboral.

Este código recoge los principales factores de riesgo del cáncer, las técnicas de diagnóstico precoz, y los signos y síntomas de alarma que con más frecuencia acompañan a la enfermedad. 
Figura 2. Competencias específicas de la Enfermería del Trabajo

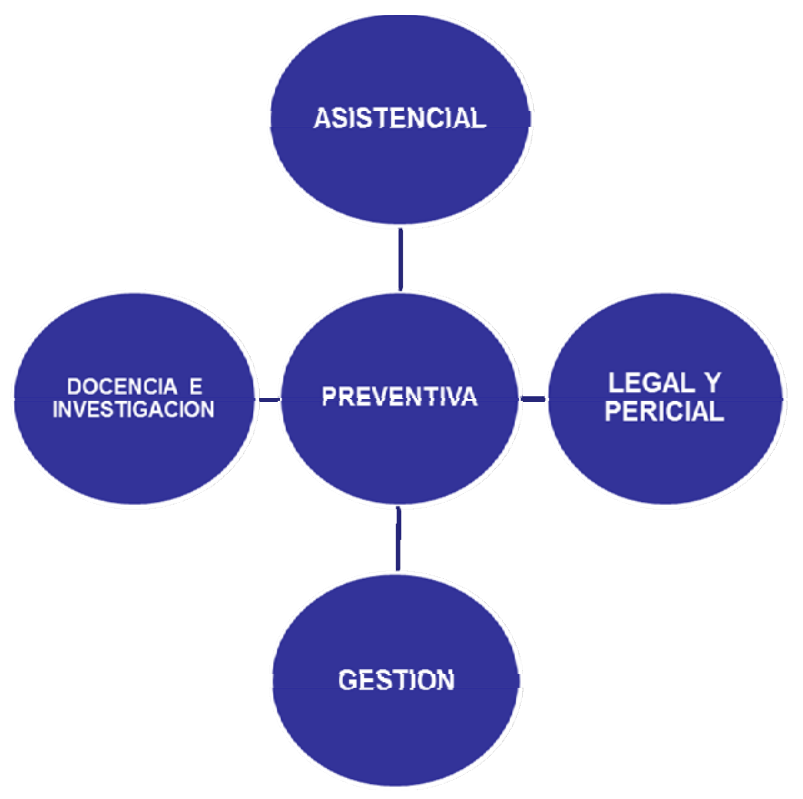

La utilización correcta de la historia clínico-laboral es clave a la hora de la aplicación del Código Europeo contra el Cáncer, pues se le atribuyen unas funciones imprescindibles, como son:

- Es la fuente más importante de recogida de datos para análisis epidemiológicos ${ }^{11}$, que permiten avanzar no solo en el conocimiento de los factores de riesgo laboral y en su prevención, sino también en el establecimiento de la relación que pueda existir entre éstos y la patología que pueda desarrollar el trabajador ${ }^{12}$, tanto aguda: accidentes de trabajo, cómo crónica: enfermedades profesionales y/o enfermedades relacionadas con el trabajo ${ }^{13}$.

- Sirve cómo testimonio documental válido en la pericia médica desde la perspectiva jurídica ${ }^{14}$.

- Puede servir como instrumento de renovación científica, al recoger datos que pueden ser utilizados con fines docentes e investigadores ${ }^{15}$.

Con la correcta utilización de la historia clínico-laboral, el especialista en Enfermería del Trabajo podrá registrar en este documento, las acciones llevadas a cabo en lo que respecta a su participación en la correcta difusión del Código Europeo Contra el Cáncer, dentro de cada una de las competencias específicas que tiene asignadas.

Dichas competencias se pueden agrupar en cinco grandes áreas de actuación, dentro de las cuales encontramos actividades a llevar a cabo, relacionadas directamente con el contenido del Código Europeo Contra el Cáncer y que se exponen a continuación:

\section{1. Área preventiva}

Dentro de esta área se incluyen las siguientes actividades:

a. Incrementar el nivel de salud de la población trabajadora, mediante acciones encaminadas a la promoción de la salud, prevención de riesgos derivados del trabajo, protección de la salud, y formación de los trabajadores.

b. Detectar precozmente los factores de riesgo derivados del trabajo, así como cualquier alteración que pueda presentarse en la población trabajadora, tanto sanos como enfermos, llevando a cabo las actividades necesarias para el control y seguimiento de los mismos, profundizando en la población especialmente sensible. 
c. Colaborar, participar, y en su caso dirigir las actividades de planificación y dirección relativas a la implantación de los planes de emergencia.

d. Realizar campañas de promoción de la salud y fomentar una cultura de salud en el ámbito de su competencia.

Las recomendaciones del Código Europeo Contra el Cáncer que podemos utilizar dentro de esta área serían las siguientes:

Recomendación 1: No fume; si fuma, abandone este hábito. Si no consigue dejar de fumar, no fume en presencia de no fumadores.

Recomendación 2: Evite la obesidad.

Para estas dos primeras recomendaciones, es importante la utilización de folletos informativos que contengan instrucciones y consejos para evitar el sobrepeso, así como información relacionada con la deshabituación tabáquica, añadiendo también la entrega de documentación que contenga tablas sencillas de ejercicios físicos a realizar por el trabajador en su tiempo libre, redactados e ilustrados de forma fácil de comprender.

Es importante demostrar disponibilidad por nuestra parte hacia el trabajador, para que en caso necesario, nos consulte cualquier tipo de duda o bien nos solicite ayuda cuando lo estime necesario.

\section{2. Área asistencial}

Las actividades a llevar a cabo en esta área son:

a. Identificar problemas de salud laboral reales o potenciales.

b. Proporcionar una atención integral a los trabajadores, ayudándoles en la recuperación de la salud y en la reincorporación al trabajo, ante todo tipo de proceso y con especial incidencia en las alteraciones de origen laboral.

c. Proponer e incentivar la continuidad de los tratamientos y cuidados proporcionados a la población trabajadora, tanto dentro como fuera de su jornada laboral, estableciendo los canales de comunicación adecuados, coordinado si es necesario con otros profesionales sanitarios.

d. Colaborar en el equipo multidisciplinar y en su caso, evaluar y documentar en el ámbito de sus competencias, la aptitud de los trabajadores para el desempeño de su trabajo, realizando los informes oportunos.

Las recomendaciones que podemos asignar dentro de esta área son la número 7 y 11.

Recomendación 7: Aplique de forma estricta la legislación destinada a prevenir cualquier exposición a sustancias carcinogénicas. Siga las instrucciones de seguridad y salud sobre el uso de estas sustancias que pueden causar cáncer. Respete las normas dictadas por las oficinas nacionales de protección radiológica.

Esta recomendación es interesante incluso desde el punto de vista del propio especialista en Enfermería del Trabajo, pues en ocasiones, en él recae la tarea de la manipulación de equipos de radiodiagnóstico dentro de esta área de asistencia al trabajador.

Las acciones dirigidas hacia los trabajadores, se encaminarían fundamentalmente en la colaboración del profesional sanitario junto con los técnicos en prevención de riesgos laborales, para la correcta vigilancia y control de los riesgos relacionados con la exposición a sustancias cancerígenas de determinadas empresas y la supervisión del cumplimiento de las medidas y normas al respecto, acciones que podrán llevarse a cabo aprovechando la asistencia prestada al trabajador cuando acuda al servicio de prevención.

Recomendación 11: Participe en programas de vacunación contra la infección por el virus de la hepatitis $B$. 
Cuando se haya reflejado en la evaluación del técnico en prevención la existencia del riesgo de contagio del virus de la hepatitis $\mathrm{B}$, el departamento médico actuará con la correspondiente ejecución del programa de vacunación contra dicho virus, ofreciendo al trabajador su correspondiente protección, asesorándole también en las medidas de seguridad y prevención necesarias, como el uso de equipos de protección individual más adecuados contra ese riesgo.

\section{3. Área, legal y pericial}

Las actividades a llevar a cabo en esta área son:

a. Valorar pericialmente, las consecuencias que tiene la patología derivada del entorno laboral para la salud humana, en particular en caso de las incapacidades.

b. Valorar pericialmente, las consecuencias que tiene la patología común que pudiera colisionar con la actividad laboral.

c. Detectar y valorar los estados de salud que sean susceptibles de cambio de puesto de trabajo.

d. Valorar pericialmente, las actuaciones sanitarias en el ámbito laboral.

e. Asesorar y orientar en la gestión y tramitación de los expedientes de incapacidad ante las entidades y los organismos competentes.

La recomendación número 6 es apropiada para llevar a cabo dentro de esta área, por la actuación importante que va ha exigir a los profesionales sanitarios de los servicios de prevención.

Recomendación 6: Tome precauciones para evitar la exposición excesiva al sol. Consulte a su médico si nota un lunar que cambia de forma, tamaño o color, algún bulto o herida que no cicatriza.

Aparte del asesoramiento e información que se debe de prestar en todo momento al trabajador, este área va a exigir al médico y enfermero del trabajo, un actitud de vigilancia y alerta importante, pues se debe de aprovechar la visita del trabajador al servicio médico durante la realización del examen de salud laboral o bien en la asistencia prestada por cualquier otro motivo, para realizar un adecuada revisión de diversos signos de alarma, fundamentalmente en toda la superficie corporal, que pudiesen sugerir el iniciar algún estudio clínico más exhaustivo.

\section{4. Área de gestión}

Las actividades a llevar a cabo en esta área son:

a. Realizar estudios, desarrollar protocolos, evaluar programas y guías de actuación para la práctica de la enfermería del trabajo.

b. Gestionar cuidados dirigidos a elevar el nivel de calidad y que permitan valorar el coste/efectividad de los mismos.

c. Trabajar y colaborar activamente en el equipo multidisciplinar de los servicios de prevención ocupando su puesto de enfermero/a especialista.

d. Gestionar y organizar los servicios de prevención de riesgos laborales en sus distintas modalidades y ámbitos.

e. Coordinar sus actuaciones con el Sistema Nacional de Salud.

f. Gestionar un sistema de registro apropiado y accesible que asegure la confidencialidad de los datos de acuerdo con lo previsto en la legislación vigente.

Las recomendaciones del Código Europeo Contra el Cáncer que podemos englobar en esta área, van ha exigir al Enfermero del Trabajo, un conocimiento actual y vigente sobre las campañas y actividades de prevención, que el sistema nacional de salud tenga dirigidas a la población en general, abarcando una serie de enfermedades cuyo riesgo 
dependerá de características personales como la edad, sexo, antecedentes familiares y determinados hábitos de vida entre otras.

Esta información debe de ser transmitida al trabajador de forma clara y detallada, pues no olvidemos que en muchas ocasiones, el personal sanitario de los servicios de prevención, constituyen el único contacto que algunos trabajadores tienen con la asistencia sanitaria, ya que no acuden de forma habitual a realizarse revisiones generales de salud.

Las recomendaciones incluidas en esta área serían:

Recomendación 8: Las mujeres a partir de los 25 años de edad deberían someterse a pruebas de detección precoz del cáncer de cuello de útero. Ello debe hacerse dentro de programas que tengan procedimientos de control de calidad de acuerdo con las "Guías Europeas para la Garantía de Calidad en el Cribado de Cáncer de Cuello de Útero”.

Recomendación 9: Las mujeres a partir de los 50 años de edad deberían someterse a pruebas de detección precoz del cáncer de mama. Ello debe hacerse dentro de programas que tengan procedimientos de control de calidad de acuerdo con las "Guías Europeas para la Garantía de Calidad en el Cribado a través de Mamografías".

Recomendación 10: Los hombres y las mujeres a partir de los 50 años de edad deberían someterse a pruebas de detección precoz de cáncer de colon. Ello debe hacerse dentro de programas que tengan integrados procedimientos de control de calidad.

La correcta divulgación de estas tres recomendaciones, son claves para colaborar en la detección y diagnostico precoz de ciertos cánceres, favoreciendo de forma importante una posible curación y mejora en la calidad de vida de las personas afectadas.

\section{5. Área de docencia e investigación}

Las actividades a llevar a cabo en esta área son:

a. Realizar comunicaciones científicas y de divulgación.

b. Informar y formar a la población trabajadora, planificando, ejecutando y evaluando programas educativos según las necesidades detectadas, de forma individualizada o en grupos.

c. Proponer e intervenir en proyectos de investigación, encaminados a mejorar continuamente la actividad profesional y con ello elevar la salud de la población trabajadora.

d. Utilizar y mejorar instrumentos que permitan medir los resultados de las intervenciones enfermeras.

La actuación de la Enfermería del Trabajo en este área, se basa en la realización de actividades de asesoramiento e información, mediante la realización de cursos, charlas y seminarios, que contengan como estructura base de sus contenidos, temas orientados a proporcionar al trabajador una mejora en ciertos hábitos de vida, así como la modificación de éstos cuando pudiesen ser nocivos para su salud.

Fundamentalmente se abarcarán temas como el consumo de alcohol y tabaco, el cumplimiento de una dieta variada y saludable, así como el fomento de la realización de una actividad física adecuada, en función de las características y necesidades de cada trabajador, utilizando para ello las siguientes recomendaciones del código:

Recomendación 3: Realice alguna actividad física vigorosa todos los días.

Recomendación 4: Aumente su ingesta diaria de frutas, verduras y hortalizas variadas: coma al menos cinco raciones al día. Limite el consumo de alimentos que contienen grasas de origen animal. 
Recomendación 5: Si bebe alcohol, ya sea cerveza, vino o bebidas de alta graduación, modere el consumo a un máximo de dos consumiciones diarias, si es hombre, o a una, si es mujer.

\section{CONCLUSIONES}

Es evidente la importancia de que el Código Europeo Contra el Cáncer no se limite a ser conocido en un reducido círculo de especialistas, sino que debe permeabilizarse a través de los profesionales y organizaciones sanitarias y de educación para la salud, para llegar así a la población general.

Queda demostrado como, dentro de todas y cada una de las áreas donde se desarrollan las competencias específicas de la Enfermería del Trabajo, tienen cabida todas las recomendaciones que forman parte del Código Europeo Contra el Cáncer, las cuales se llevarán a cabo con la ejecución de las actividades asignadas a cada área.

Los últimos estudios relacionados con el cáncer, ponen de manifiesto la importancia que tiene una adecuada actuación por parte del personal médico en la detección y diagnóstico precoz, favoreciendo de forma clave la curación y calidad de vida de los individuos afectados. Dicha actuación debe de realizarse también en los servicios de prevención, favoreciendo esta detección y diagnostico precoz en la población trabajadora.

\section{REFERENCIAS BIBLIOGRÁFICAS}

1. Benavides FG, Castejón J, Gimeno D, Porta M, Mestres J, Simonet P. Certification of occupational diseases as common diseases in a primary health care setting. Am J Ind Med. 2005; 47: 176-80.

2. Kogevinas M., Rodríguez Suárez M., Adonina Tardón S., Cáncer Laboral en España. Institut Municipal d'Investigació Médica. Barcelona; 2005.

3. P. Sanz-Gallén, J. Izquierdo, A. Prat Marín. Manual de Salud Laboral, Cáncer laboral. Barcelona: Springer - Verlag Ibérica; 2001: 330-338.

4. García Gómez M, Kogeviñas M. Estimación de la mortalidad por cáncer laboral y de la exposición a cancerígenos en el lugar de trabajo en España en los años 90. Gac Sanit. 1996; 10:143-151.

5. Ley 14/1986, de 25 de abril, General de Sanidad. BOE número 102 de 29 de Abril de 1986: 15207-15224.

6. Ley 31/1995, de 8 de Noviembre, de Prevención de Riesgos Laborales. BOE núm. 269, de 10 de Noviembre de 1995 .

7. Real Decreto 665/1997, de 12 de mayo, sobre la protección de los trabajadores contra los riesgos relacionados con la exposición a agentes cancerígenos durante el trabajo, con las modificaciones introducidas por el Real Decreto 1124/2000 y el Real Decreto 349/2003

8. Estrategia española de Seguridad y salud en el Trabajo (2007-2012). URL:http://www.isciii.es/htdocs/ centros/medicinadeltrabajo/ultima_hora/EESST.pdf Consultado: 30/06/2012

9. Gonzalez CA, Agudo A. Occupational cancer in Spain.Environ Health Perspect. 1999; 107: $273-277$.

10. Orden SAS/1348/2009, de 6 de mayo, por la que se aprueba y publica el programa formativo de la especialidad de Enfermería del Trabajo, BOE núm. 129, de 28 de Mayo.

11. Real decreto. 39/97 de 17 de enero, por el que se aprueba el Reglamento de los Servicios de Prevención. BOE núm. 27 de 31 enero.

12. Frank, A.: Approach to the patient with an occupational or environmental illness: Occup Environ Med $2000 ; 27: 877-893$.

13. Gomero Cuadra, R. Llaypesan, C.: La historia médico-ocupacional cómo herramienta de diagnóstico. Rev Med Hered 2005; 16(3):199-201.

14. Cantero-Rivas R. La historia clínica; de documento médico a documento médico-legal. Todo Hospital 1997;142: 43-9.

15. Castellano Arroyo, M. Villanueva Cañadas, E.: Derecho médico relacionado con la Medicina del Trabajo. En: Gil Hernández, F.: Tratado de Medicina del Trabajo. Barcelona: Masson, 2005. 